\title{
A NOTE ON THE REPLICAS OF NILPOTENT MATRICES
}

\author{
HSIO-FU TUAN
}

In a recent paper, ${ }^{1}$ Chevalley proved the following theorem:

(A) If $Z$ is a nilpotent matrix over a field $K$ of characteristic 0 , the only replicas $Z^{\prime}$ of $Z$ are the matrices $Z^{\prime}=t Z, t \in K .^{2}$

For the proof of (A), he made use of a particular case of a theorem due to Ado and gave a proof for the results which he needed. In the present note, we shall give a direct simple proof of (A) and we shall in fact deduce it as an immediate consequence of the stronger theorem:

(B) If $Z$ and $Z^{\prime}$ are two nilpotent matrices over a field $K$ of characteristic 0 , and if $q(x)$ and $r(x)$ are two polynomials with coefficients in $K$ and without constant terms such that $Z^{\prime}=q(Z)$ and $Z_{0,2}^{\prime}=r\left(Z_{0,2}\right)$, then $Z^{\prime}=t Z, t \in K$.

We shall later establish corresponding results for fields $K$ of prime characteristics, to be stated as theorems (C) and (D).

That (A) is implied by (B) follows immediately from the fact that if $Z^{\prime}$ is a replica of $Z$, then $Z_{r, s}^{\prime}=p_{r, s}\left(Z_{r, s}\right)$, where $p_{r, s}(x)$ are polynomials in $K$ without constant terms. ${ }^{3}$

For the proof of (B), let $n$ be the degree of $Z$ and $Z^{\prime}$ and let $m$ be the least nonnegative integer such that $Z^{m+1}=0$. Clearly $0 \leqq m \leqq n-1$. The case $Z=0$ is trivial; we can therefore assume $1 \leqq m \leqq n-1$. Let also $l$ be the least nonnegative integer such that $\left(Z_{0,2}\right)^{l+1}=0$. Clearly $Z_{0,2}$ is nilpotent and $1 \leqq l \leqq n^{2}-1$. We shall see that $m \leqq l \leqq 2 m$.

The matrix $Z$ can be transformed by an $(n, n)$ matrix $T$ with coefficients in the algebraic closure $\bar{K}$ of $K$ into the following form:

$$
Z_{1}=T^{-1} Z T=\left(\begin{array}{ccccc}
0 & & & \\
z_{1} & 0 & & \\
& \ddots & \ddots & \\
& \ddots & 0 & \\
& & z_{n-1} & 0
\end{array}\right),
$$

where $z_{1}, \cdots, z_{n-1}$ are zeros and ones and not all żeros. Then for

Received by the editors July 17, 1944.

${ }^{1}$ Claude Chevalley, On a kind of new relationship between matrices, Amer. J. Math. vol. 65 (1943) pp. 521-531.

2 Theorem 6, p. 530, loc. cit.

${ }^{3}$ Lemma 4, p. 529, loc. cit. 
any integer $i, 1 \leqq i \leqq m$, we have

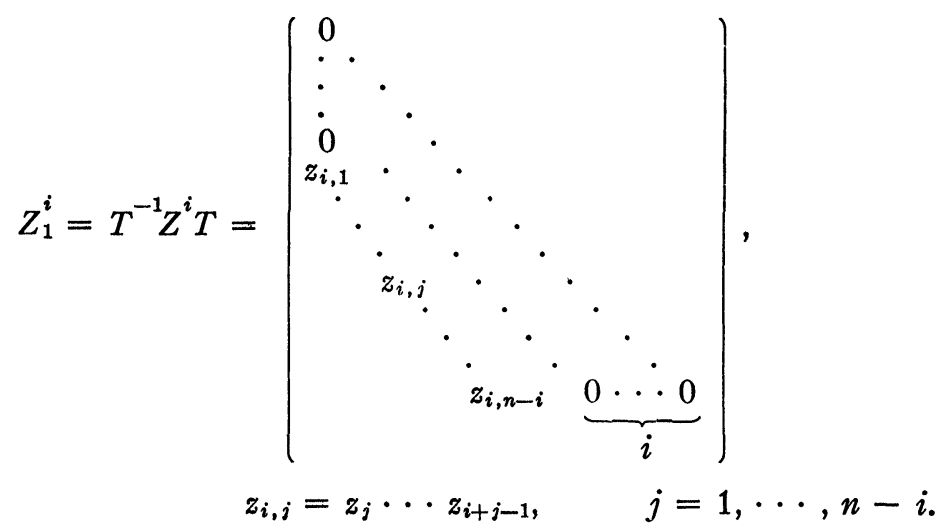

Hence if we write

$$
q(x)=q_{1} x+\cdots+q_{m} x^{m}, q_{i} \in K, \quad i=1, \cdots, m,
$$

we have

$$
Z_{1}^{\prime}=T^{-1} Z^{\prime} T=T^{-1} q(Z) T=q\left(T^{-1} Z T\right)=q\left(Z_{1}\right) .
$$

Denoting the $(n, n)$ identity matrix by $E$, we have then ${ }^{4}$

$$
\left(Z_{1}\right)_{0,2}=Z_{1} \oplus Z_{1}=Z_{1} \otimes E+E \otimes Z_{1}
$$

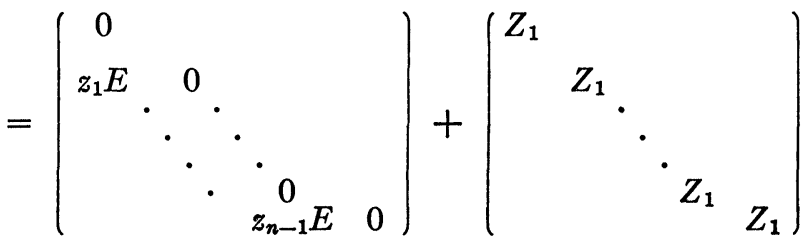

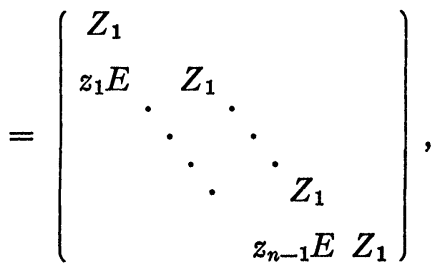

hence for any positive integer $i, 5$

4 We define $A \otimes B=\left(a_{i j}\right) \otimes B=\left(a_{i j} B\right)$. Observe that $(A \otimes B)(C \otimes D)=A B \otimes C D$. The heavy cross and heavy plus used in Chevalley's paper are here replaced by $\otimes$ and $\oplus$.

${ }^{5}$ In the following, * denotes the terms in which we are not interested. 


$$
\begin{aligned}
\left(\left(Z_{1}\right)_{0,2}\right)^{i} & =\left(Z_{1} \otimes E+E \otimes Z_{1}\right)^{i} \\
& =Z_{1} \otimes E+\sum_{j=1}^{i-1} C_{i, j} Z_{1}^{i-i} \otimes Z_{1}^{j}+E \otimes Z_{1}^{i}
\end{aligned}
$$

$$
=\left(\begin{array}{ccccc}
Z_{1}^{i} & & & & \\
& Z_{1}^{i} & & & \\
& & \ddots & & \\
& & \cdot & Z_{1}^{i} & \\
& & & & Z_{1}^{i}
\end{array}\right)
$$

( $C_{i, j}$ being the binomial coefficients), and therefore $m \leqq l \leqq 2 m$. We may write

$$
r(x)=r_{1} x+\cdots+r_{l} x^{l}, r_{i} \in K, \quad i=1, \cdots, l,
$$

and then

$$
\begin{aligned}
\left(Z_{1}^{\prime}\right)_{0,2} & =Z_{1}^{\prime} \oplus Z_{1}^{\prime}=Z_{1}^{\prime} \otimes E+E \otimes Z_{1}^{\prime} \\
& =T^{-1} Z^{\prime} T \otimes T^{-1} E T+T^{-1} E T \otimes T^{-1} Z^{\prime} T \\
& =\left(T^{-1} \otimes T^{-1}\right)\left(Z^{\prime} \otimes E+E \otimes Z^{\prime}\right)(T \otimes T) \\
& =(T \otimes T)^{-1} Z_{0,2}^{\prime}(T \otimes T)=(T \otimes T)^{-1} r\left(Z_{0,2}\right)(T \otimes T) \\
& =r\left((T \otimes T)^{-1} Z_{0,2}(T \otimes T)\right)=r\left(\left(Z_{1}\right)_{0,2}\right) .
\end{aligned}
$$

Consequently the same relations originally assumed for $Z$ and $Z^{\prime}$ now hold for $Z_{1}$ and $Z_{1}^{\prime}$. For simplicity in notations, we shall now just consider $Z$ and $Z^{\prime}$ for $Z_{1}$ and $Z_{1}^{\prime}$ in the related formulas (1)i, (3), $(4)_{i},(6)$.

Now, on the one hand,

$$
\begin{aligned}
& Z_{0,2}^{\prime}=Z^{\prime} \oplus Z^{\prime}=Z^{\prime} \otimes E+E \otimes Z^{\prime}=q(Z) \otimes E+E \otimes q(Z) \\
& =E \otimes q(Z)+\left(q_{1} Z+\cdots+q_{m} Z^{m}\right) \otimes E \\
& =E \otimes q(Z)+Z \otimes q_{1} E+\cdots+Z^{m} \otimes q_{m} E
\end{aligned}
$$

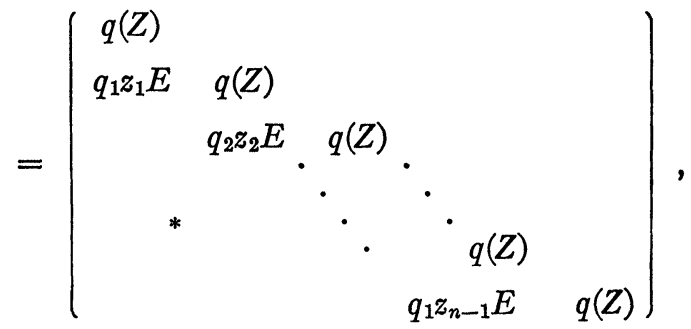

while, on the other hand, 


$$
\begin{aligned}
& Z_{0,2}^{\prime}=r\left(Z_{0,2}\right)=r_{1} Z_{0,2}+r_{2}\left(Z_{0,2}\right)^{2}+\cdots+r_{l}\left(Z_{0,2}\right)^{l} \\
& =r_{1}(Z \oplus Z)+r_{2}(Z \oplus Z)^{2}+\cdots+r_{l}(Z \oplus Z)^{l} \\
& =r_{1}(Z \otimes E+E \otimes Z)+r_{2}\left(Z^{2} \otimes E+2 Z \otimes Z+E \otimes Z^{2}\right) \\
& +\cdots+r_{l}\left(Z^{l} \otimes E+\sum_{i=1}^{l-1} Z^{l-i} \otimes Z^{i}+E \otimes Z^{l}\right) \\
& =E \otimes\left(r_{1} Z+r_{2} Z^{2}+\cdots+r_{l} Z^{l}\right) \\
& +Z \otimes\left(r_{1} E+2 r_{2} Z+\cdots+\operatorname{lr}_{l} Z^{l-1}\right) \\
& +\cdots+Z^{l} \otimes r_{l} E \\
& =E \otimes r(Z)+Z \otimes r^{\prime}(Z)+Z^{2} \otimes(1 / 2) r^{\prime \prime}(Z) \\
& +\cdots+Z^{l} \otimes(1 / l !) r^{(l)}(Z)
\end{aligned}
$$

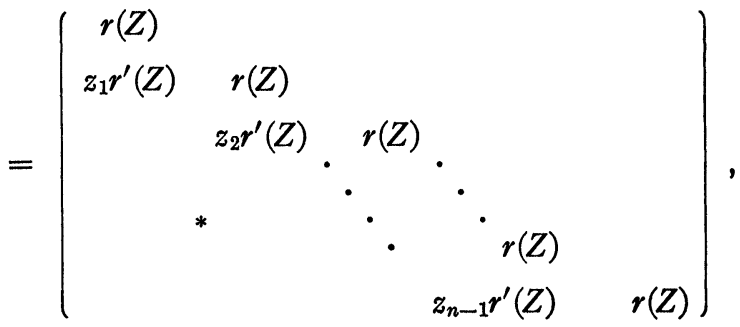

where $r^{\prime}(x), r^{\prime \prime}(x), \cdots, r^{(l)}(x)$ are the successive derivatives of $r(x)$.

In (7) and (8), comparing the terms (which are $(n, n)$ matrices) on the main diagonal and on the first parallel just below, we obtain

$$
\begin{aligned}
q(Z) & =r(Z), \\
q_{1} z_{i} E & =z_{i} r^{\prime}(Z) ; \quad i=1, \cdots, n-1 .
\end{aligned}
$$

(9) gives

and hence

$$
q_{1} Z+\cdots+q_{m} Z^{m}=r_{1} Z+\cdots+r_{l} Z^{l}, \quad l \geqq m,
$$

$$
q_{j}=r_{j}, \quad j=1, \cdots, m .
$$

(10) gives

$$
q_{1} z_{i} E=z_{i}\left(r_{1} E+2 r_{2} Z+\cdots+\operatorname{lr}_{l} Z^{l-1}\right), \quad i=1, \cdots, n-1,
$$

by (11),

$$
z_{i}\left(2 q_{2} E+\cdots+m q_{m} Z^{m-1}+\cdots\right)=0, \quad i=1, \cdots, n-1,
$$

as not all $z_{i}$ (for $i=1, \cdots, n-1$ ) are zero, hence

$$
2 q_{2} E+\cdots+m q_{m} Z^{m-1}+\cdots=0,
$$


and consequently

$$
2 q_{2}=\cdots=m q_{m}=0 .
$$

Now $K$ is of characteristic 0 ; we can therefore conclude

$$
q_{2}=\cdots=q_{m}=0 \text {. }
$$

It then follows that

$$
Z^{\prime}=q_{1} Z=t Z
$$

with $q_{1}=t \in K$, as is to be proved.

Let us now suppose that $K$ is of prime characteristic, say $p$. If $p>m$, which is certainly the case if $p \geqq n$, then we can still infer (13) from (12) and hence still have (14) as before. For the cases $p \leqq m$, from (12) we can only infer that

$$
q_{i}=0, \quad 2 \leqq i \leqq m, \quad p \nmid i,
$$

and hence we can only conclude that $Z^{\prime}$ is necessarily of the form

$$
Z^{\prime}=q_{1} Z+q_{p} Z^{p}+q_{2 p} Z^{2 p}+\cdots+q_{m^{\prime} p} Z^{m^{\prime} p},
$$

where $m^{\prime}=[m / p]$, the largest integer not greater than $m / p$. In fact, the conclusions (13) and (14) are then no longer true in general.

We shall first prove that $Z^{\prime}=Z^{\alpha}$ with $\alpha=p^{a}$ ( $a$ being any nonnegative integer) is a replica of $Z$. This follows from the facts:

$$
\begin{aligned}
& Z_{r, 8}^{\prime}=\left(Z^{\alpha}\right)_{r, 8}=\underbrace{\left(Z^{\alpha}\right) \oplus \cdots \oplus\left(Z^{\alpha}\right)}_{r} \oplus \underbrace{\left(Z^{\alpha}\right) \oplus \cdots \oplus\left(Z^{\alpha}\right)}_{s} \\
& =\left(-{ }^{t} Z^{\alpha}\right) \oplus \cdots \oplus\left(-{ }^{t} Z^{\alpha}\right) \oplus Z^{\alpha} \oplus \cdots \oplus Z^{\alpha} \\
& =-\left({ }^{t} Z^{\alpha}\right) \otimes \cdots \otimes E \otimes E \otimes \cdots \otimes E-\cdots \\
& -E \otimes \cdots \otimes\left({ }^{t} Z^{\alpha}\right) \otimes E \otimes \cdots \otimes E \\
& +E \otimes \cdots \otimes E \otimes Z^{\alpha} \otimes \cdots \otimes E+\cdots \\
& +E \otimes \cdots \otimes E \otimes E \otimes \cdots \otimes Z^{\alpha} \\
& =\left(-{ }^{t} Z \otimes \cdots \otimes E \otimes E \otimes \cdots \otimes E-\cdots\right. \\
& -E \otimes \cdots \otimes t Z \otimes E \otimes \cdots \otimes E \\
& +E \otimes \cdots \otimes E \otimes Z \otimes \cdots \otimes E+\cdots \\
& +E \otimes \cdots \otimes E \otimes E \otimes \cdots \otimes Z)^{\alpha} \\
& =\left(Z_{r, 8}\right)^{\alpha} \text {, }
\end{aligned}
$$

because $(-1)^{\alpha}= \pm 1 \equiv-1(\bmod p)$ for $p=2$ and $(-1)^{\alpha}=-1$ for $p \neq 2$. 
We shall now prove that conversely if $Z^{\prime}=q(Z)=\sum_{i=1}^{m} q_{i} Z^{i}$ with $q_{i} \in K$ is a replica of $Z$, then we have

(18) $\quad Z^{\prime}=q(Z)=\sum_{j=1}^{m_{0}} q_{i(j)} Z^{i(j)}, \quad i(j)=p^{j}, \quad i\left(m_{0}\right) \leqq m<i\left(m_{0}+1\right)$.

We shall show more strongly that only then $Z_{1,1}^{\prime}=s\left(Z_{1,1}\right)$, where $s(x)$ is a polynomial with coefficients in $K$ and without the constant term.

We can assume that $Z$ is of the form (1); because as before,

$$
\left(Z_{1}^{\prime}\right)_{1,1}=\left(T^{-1} Z^{\prime} T\right)_{1,1}=\left({ }^{t} T^{-1} \otimes T\right)^{-1} Z_{1,1}^{\prime}\left({ }^{t} T^{-1} \otimes T\right),
$$

hence $Z_{1,1}^{\prime}=r\left(Z_{1,1}\right)$ implies also

$$
\begin{aligned}
\left(Z_{1}^{\prime}\right)_{1,1} & =\left({ }^{t} T^{-1} \otimes T\right)^{-1} s\left(Z_{1,1}\right)\left({ }^{t} T^{-1} \otimes T\right) \\
& =s\left(\left({ }^{t} T^{-1} \otimes T\right)^{-1} Z_{1,1}\left({ }^{t} T^{-1} \otimes T\right)\right)=s\left(\left(Z_{1}\right)_{1,1}\right) .
\end{aligned}
$$

Then, for any positive integer $i, 1 \leqq i \leqq m$, we have

$(20)_{i}$

$$
\begin{aligned}
& \left(Z^{\prime}\right)^{i}=\left(-{ }^{t} Z\right)^{i}=(-1)^{i}{ }^{t} Z_{\imath} \\
& =\left(\begin{array}{cccccc}
0 \cdots & \cdots & z_{i, 1} & & & \\
\ddots & \ddots & \ddots & & \\
& \ddots & \ddots & z_{i, j} & \\
& & \ddots & \ddots & \ddots & z_{i, n-i} \\
& & & \ddots & \cdot & 0 \\
& & & & \ddots & \vdots \\
& & & & & 0
\end{array}\right), \\
& z_{i, j}=(-1)^{i} z_{j} \cdots z_{i+j-1}, \quad j=1, \cdots, n-i,
\end{aligned}
$$

and further

$$
\begin{aligned}
& \left(Z_{1,1}\right)^{i}=\left(Z^{\prime} \oplus Z\right)^{i}=\left(Z^{\prime} \otimes E+E \otimes Z\right)^{i}=\left(-{ }^{t} Z \otimes E+E \otimes Z\right)^{i} \\
& =(-1)^{i}{ }^{i} Z^{i} \otimes E+\sum_{j=1}^{i-1}(-1)^{i-i} C_{i, j} Z^{i-j} \otimes Z^{j}+E \otimes Z^{i}
\end{aligned}
$$

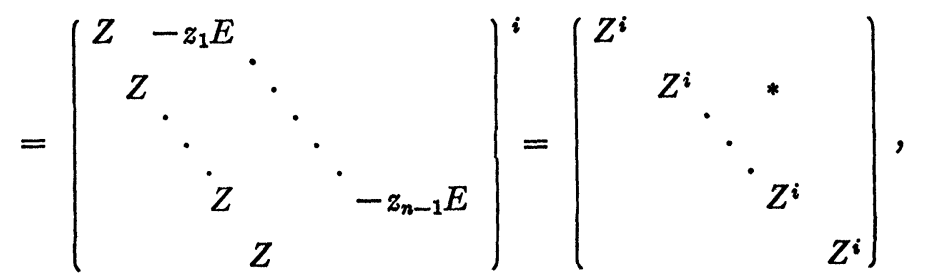


hence it follows as before that the least nonnegative integer $k$ such that $\left(Z_{1,1}\right)^{k+1}=0$ satisfies $m \leqq k \leqq 2 m$. We can therefore write

$$
\begin{aligned}
s(x) & =s_{1} x+\cdots+s_{k} x^{k} \quad\left(s_{i} \in K\right) \\
& =\left(s_{1} x+\cdots+s_{m} x^{m}\right)+\left(s_{m+1} x^{m+1}+\cdots+s_{k} x^{k}\right) \\
& =s^{\prime}(x)+s^{\prime \prime}(x) .
\end{aligned}
$$

We have

$$
\begin{aligned}
Z_{1,1}^{\prime} & =Z^{\prime} \oplus Z=\left(-{ }^{t} Z^{\prime}\right) \oplus Z=-{ }^{t} Z^{\prime} \otimes E+E \otimes Z^{\prime} \\
& =-q\left({ }^{t} Z\right) \otimes E+E \otimes q(Z) \\
& =E \otimes q(Z)+\sum_{j=1}^{m} t^{j} \otimes\left(-q_{j} E\right),
\end{aligned}
$$

while, on the other hand,

$$
\begin{aligned}
Z_{1,1}^{\prime} & =\sum_{i=1}^{k} s_{i}\left(Z_{1,1}\right)^{i}=\sum_{i=1}^{k} s_{i}\left(-{ }^{t} Z \otimes E+E \otimes Z\right)^{i} \\
& =\sum_{i=1}^{k} s_{i} \sum_{j=\max (0, i-m)}^{\min (i, m)} C_{i, j}\left(-{ }^{t} Z\right)^{j} \otimes Z^{i-j} \\
& =\sum_{j=0}^{m} t^{j} \otimes(-1)^{i}\left(\sum_{i=\max (1, j)}^{\min (k, m+j)} s_{i} C_{i, j} Z^{i-j}\right) \\
& =E \otimes \sum_{i=1}^{m} s_{i} Z_{i}+\sum_{j=1^{\prime}}^{m} Z^{j} \otimes(-1)^{j}\left(\sum_{i=j}^{\min (k, m+j)} s_{i} C_{i, j} Z^{i-j}\right) \\
& =E \otimes s^{\prime}(Z)+\sum_{j=1}^{m} t^{j} \otimes s_{j}(Z),
\end{aligned}
$$

where $s_{j}(x)$ are polynomials with coefficients in $K$ for $j=1, \cdots, m$ (observe $\max (0, i-m) \leqq \min (i, k-m) \leqq \min (i, m))$. Writing the matrix $Z_{1,1}^{\prime}$ as given by (23) and (24) in the form of two compound $(n, n)$ matrices whose elements are again $(n, n)$ matrices and comparing the terms on their main diagonals and on their $m$ first parallels above the main diagonals, we can then conclude that first

$$
\begin{aligned}
q(Z) & =\sum_{i=1}^{m} q_{i} Z^{i}=\sum_{i=1}^{m} s_{i} Z^{i}=s(Z), & \\
q_{i} & =s_{i}, & i=1, \cdots, m ;
\end{aligned}
$$

and that also

$$
z_{j, h}\left(-q_{j} E\right)=z_{j, h} s_{j}(Z), \quad j=1, \cdots, m, h=1, \cdots, n-j,
$$


as $Z^{\prime}{ }^{\prime} \neq 0$ for $j=1, \cdots, m$, so for each $j$ we have at least one $h$, say $h(j)$ (=one of $1, \cdots, n-j$ ), such that $z_{j, h(j)} \neq 0$, then $z_{j, h(j)}= \pm 1$, and hence

$$
\begin{aligned}
-q_{j} E & =s_{j}(Z)=(-1)^{j} \sum_{i=j}^{\min (k, m+j)} s_{i} C_{i, j} Z^{i-j} \\
& =(-1)^{j^{j}} s_{j} E+(-1)^{j} \sum_{i=j+1}^{\min (k, m+j)} s_{i} C_{i, j} Z^{i-j,}, \\
0 & =\left(1+(-1)^{j}\right) s_{j} E+(-1)^{j} \sum_{i=j+1}^{\min (k, m+j)} s_{i} C_{i, j} Z^{i-j}, \\
\left(1+(-1)^{j}\right) s_{j}=0, \quad s_{i} C_{i, j}=0, \quad j=1, \cdots, m ; & \quad i=j+1, \cdots, \min (k, m+j)^{6}
\end{aligned}
$$

(observe $\min (k, m+j) \geqq m)$, in particular

$$
s_{i} C_{i, j}=0, \quad i=2, \cdots, m ; j=1, \cdots i-1 .
$$

Now it is easily seen that if $i=p^{i i^{\prime}}$ with $p \nmid i^{\prime}$, then $p \nmid C_{i, i / i^{\prime},{ }^{7} \text { hence }}$ it follows immediately that if $i^{\prime} \neq 1$, namely, $i \neq p^{j}$ for $j=1, \cdots, m_{0}$ with $p^{m_{0}} \leqq m<p^{m_{0}+1}$, then $q_{i}=s_{i}=0$ for $i=2, \cdots, m$, as was to be proved.

Summarizing our results for fields $K$ of characteristic $p \neq 0$, we have the following theorems:

(C) If $Z$ and $Z^{\prime}$ are two nilpotent matrices over a field $K$ of characteristic $p \neq 0$, and if $q(x), r(x)$ and $s(x)$ are three polynomials with coeffcients in $K$ and without constant terms such that $Z^{\prime}=q(Z), Z_{0,2}^{\prime}=r\left(Z_{0,2}\right)$ and $Z_{1,1}^{\prime}=s\left(Z_{1,1}\right)$, then we have

$$
Z^{\prime}=q(Z)=\sum_{j=0}^{m_{0}} t_{j} Z^{i(j)}, \quad i(j)=p^{j}, \quad t_{j} \in K .
$$

(D) If $Z$ is a nilpotent matrix over a field $K$ of characteristic $p \neq 0$, the only replicas $Z^{\prime}$ of $Z$ are the matrices (30).

Princeton University

B If $k=m$, then for $j=m$, there is no $i, j+1 \leqq i \leqq \min (k, m+j)$.

${ }^{7}$ Consider $p^{i}=p^{i} \cdots 1$ and $i \cdots\left(i-p^{i}+1\right)=\left(p^{i}+p^{i}\left(i^{\prime}-1\right)\right) \cdots\left(1+p^{i}\left(i^{\prime}-1\right)\right)$. As $p^{v} \| u$ (namely $p^{v} \mid u$ and $p^{v+1}\left\langle u\right.$ ) for $u=p^{i} ; \cdots, 1$ implies $p^{v} \|\left(u+p^{j}\left(i^{\prime}-1\right)\right.$ ) because $v \leqq j$, so $p \nmid C_{i, i / i^{\prime}}$. 